\title{
Immune checkpoint inhibitors and non-small-cell lung cancer management: 2018 update
}

\author{
Omar Abdel-Rahman*,1 \& Don Morris ${ }^{1}$ \\ ${ }^{1}$ Department of Oncology, University of Calgary, Tom Baker Cancer Centre, Calgary, Alberta, Canada \\ *Author for correspondence: Tel.: +1 403918 9779; omar.abdelsalam@ahs.ca
}
" Recently, immune response evaluation criteria in solid tumor was proposed and it advocates for a different approach for response evaluation that takes into consideration the well-recorded phenomenon of pseudoprogression among solid tumor patients treated with these agents"

First draft submitted: 17 October 2017; Accepted for publication: 6 November 2018; Published online: 16 January 2019

Keywords: immunotherapy $\bullet$ NSCLC • outcome • prognosis • survival

Immune checkpoint inhibitors provided one of the most important breakthroughs in the management of solid tumors in the past decade [1,2]. Their effect was particularly relevant among some hard-to-treat malignancies including non-small-cell lung cancer (NSCLC), malignant melanoma and renal cell carcinoma among others. The pace of clinical developments in NSCLC, in particular, has been quite revolutionary in the past 4 years [3].

Among previously treated NSCLC patients, four randomized Phase III studies have demonstrated an overall survival (OS) benefit for the three PD-L1 inhibitors (pembrolizumab, nivolumab and atezolizumab) compared with docetaxel monotherapy [4-7]. Based on these landmark studies, the three agents were granted regulatory approval for use in this indication. However, because of the difference in eligibility criteria for these trials, the conditions for regulatory approval were different. While pembrolizumab approval was restricted to patients with PD-L1 > $1 \%$, nivolumab and atezolizumab approvals were independent of PD-L1 status. Additional systematic reviews have shown that a number of factors - in addition to PD-L1 status - can affect survival benefit from these agents. These possibly include smoking status and EGFR status [8,9]. These findings have affected later design and conduct of trials of PD-L1 inhibitors in previously untreated NSCLC.

After 1 year, two Phase III trials evaluated the impact of each of pembrolizumab and nivolumab on outcomes of previously untreated NSCLC (KeyNote-024 and Checkmate-026) [10,11]. For KeyNote-024 study, inclusion was restricted to patients with PD-L1 $\geq 50 \%$ without EGFR or ALK mutations; while for Checkmate-024 study, inclusion was restricted to patients with PD-L1 $>1 \%$. While KeyNote-024 study was a clear success story with OS improvement compared with platinum-based doublet chemotherapy (hazard ratio for death: 0.60; 95\% CI: 0.41$0.89 ; \mathrm{p}=0.005)$, Checkmate-026 was not as successful without demonstrated overall or progression-free survival (PFS) benefit.

These findings from the two Phase III studies have ignited intense debate about proper optimization and patient selection in the setting of immune checkpoint inhibitors for advanced NSCLC. Lack of standardization of PD-L1 testing used for each agent and possible inter- and intra-observer variability in reading this biomarker impact subsequent therapeutic decision-making.

A number of strategies were explored in the year 2018 in order to overcome this potential drawback. These are detailed in the following sections.

\section{Tumor mutational burden as an alternative biomarker}

Tumor mutational burden was long claimed to play an important role in predicting outcomes of solid tumor patients treated with immune checkpoint inhibitors [12]. Recently, Checkmate-227 study elaborated on this principle and it has shown that among patients with NSCLC and a high tumor mutational burden (irrespective of PD-L1 expression

Future $\because$ Medicine 
level), PFS was significantly longer with first-line nivolumab plus ipilimumab compared with chemotherapy [13]. These results suggest a potential role for immunotherapy-immunotherapy combinations in NSCLC as well as a potential role for tumor mutational burden in predicting the outcomes of NSCLC treated with immune checkpoint inhibitors.

More recently, tumor mutational burden was compared with PD-L1 as a predictive marker for patients with nonsquamous NSCLC receiving second line PD-(L)1 inhibitors. In this study, tumor mutational burden and PD-L1 were not correlated and high tumor mutational burden was associated with improved response rate, PFS and OS [14]. Additionally, a recent study reported in an abstract form suggested that blood-based tumor mutational burden test might be used to predict the outcomes of patients receiving first line PD-L1 inhibitors [15].

\section{Chemotherapy-immunotherapy combination}

The year 2018 has shown the establishment of a number of chemotherapy/immunotherapy combinations in the first line treatment of NSCLC. The first study, which was presented in AACR meeting in 2018 and published in NEJM, has shown that among patients with nonsquamous NSCLC (without EGFR or ALK mutations), pemetrexed-platinum-pembrolizumab combination improved survival compared with chemotherapy alone [16]. This study included patients regardless of their PD-L1 status.

A similar study in advanced squamous NSCLC (KeyNote-407) evaluated chemotherapy (carboplatin-paclitaxelnab-paclitaxel) with or without pembrolizumab. There was significant overall and PFS improvement with immunochemotherapy combination in this setting [17]. This study also included patients regardless of their PD-L1 status.

A third study evaluated combination of carboplatin-paclitaxel-bevacizumab with or without atezolizumab in the first line treatment of nonsquamous NSCLC (IMpower150). Immunochemotherapy combination was associated with improved overall and PFS compared with chemotherapy alone [18]. Notably, this study included patients with EGFR/ALK mutations and the benefit was observed regardless of PD-L1, ALK or EGFR statuses.

Two additional Phase III studies of chemotherapy/immunotherapy combination were reported in an abstract form. These include IMpower130 and IMpower132. IMpower130 evaluated carboplatin-nab-paclitaxel with or without atezolizumab among patients with nonsqauamous NSCLC. Among randomized patients without EGFR or ALK mutations, atezolizumab + chemotherapy combination improved overall and PFS [19]. IMpower132 study evaluated platinum-pemetrexed combination with or without atezolizumab among patients with nonsquamous NSCLC. While atezolizumab + chemotherapy combination improved PFS in this study, OS data are not yet mature [20].

At the time of reporting of these studies, another international Phase III trial was reported which compared pembrolizumab monotherapy versus platinum-based chemotherapy as first-line therapy for advanced NSCLC and a PD-L1 $\geq 1 \%$ (no EGFR/ALK mutation; KeyNote-042) [21]. In the overall cohort, pembrolizumab monotherapy has better overall and PFS compared with chemotherapy. However, on exploratory analysis, the survival benefit was restricted to the sub-category of patients with PD-L1 $\geq 50 \%$. Thus, this study essentially confirms the results of previous studies but does not change the standard practice.

\section{Moving PD-L1 blockade into early NSCLC}

The positive results from PD-L1 inhibitors in advanced disease encouraged the evaluation of these agents in earlier stages of the disease. Last year, durvalumab maintenance treatment following chemoradiotherapy for unresectable stage III NSCLC has shown significant survival improvement [22]. It has thus received regulatory approval as a standard of care in this indication. Subsequent secondary analysis of the PACIFIC study suggested that durvalumab provided clinical benefit in this group of patients regardless of time from radiotherapy to randomization. There was also no apparent difference in adverse events, including high grade adverse events, in patients who started durvalumab treatment within 2 weeks from the end of radiotherapy versus later than 2 weeks [23].

This year, we have also observed the first positive results for PD-L1 inhibitors in the neoadjuvant setting for resectable lung cancer. In this study, neoadjuvant nivolumab was associated with few side effects, did not delay surgery, and induced a major pathological response in $45 \%$ of resected tumors [24]. The results of additional adjuvant studies for PD-L1 inhibitors in NSCLC are awaited. Another study reported recently in an abstract form has shown that neoadjuvant nivolumab/ipilimumab was associated with a major pathological response in $31 \%$ of included patients [25]. 


\section{Safety considerations}

The peculiar mechanism of action of immune checkpoint inhibitors comes at a price of a higher risk of a new class of adverse events (immune-related adverse events). This class of adverse events is diverse and might involve almost any organ in the body [26,27]. Of particular interest among patients with lung cancer is the risk of immune-related pneumonitis as the clinical of pneumonitis might be confused with the clinical presentation of disease progression [28]. These well-known safety considerations might be further complicated by the newly adopted regimens combining chemotherapy + immune checkpoint inhibitors. Thus, clinical judgment needs to be meticulously employed before embarking patients on such combination regimens.

\section{New response evaluation criteria}

Immune checkpoint inhibitors are well known for their unconventional patterns of benefit/response [29]. Thus, it is becoming increasingly known that traditional response evaluation criteria in solid tumors do not represent a good representative model of benefit for patients receiving immune checkpoint inhibitors.

Recently, immune response evaluation criteria in solid tumor was proposed and it advocates for a different approach for response evaluation that takes into consideration the well-recorded phenomenon of pseudoprogression among solid tumor patients treated with these agents [30]. This is particularly relevant among lung cancer patients treated with these agents because of the commonly encountered difficulty in determining patients who really benefit from these treatments.

\section{New dosing recommendations}

Previously, pembrolizuab and nivolumab dose were calculated based on body weight. Based on previous analyses which suggested efficacy and toxicity equivalence between different dose schedules of pembrolizumab and nivolumab [31], the FDA recently approved a fixed-dose strategy for both drugs (in order to facilitate treatment administration and avoid confusion). Likewise, instead of a biweekly schedule of nivolumab, a 4-weekly schedule was recently approved in order to improve the logistics of nivolumab administration [32].

\section{Summary \& conclusion}

- Based on the available Phase III evidence, the following treatment scenarios/treatment options for patients with metastatic NSCLC and no driver mutations might be postulated.

1. PD-(L) 1 expression $\geq 50 \%$ : pembrolizumab single agent seems to be a reasonable option based on KeyNote-024 data.

2. Higher tumor mutational buren: nivolumab-ipilimumab combination seems to be a preferred treatment option based on Checkmate-227 study.

3. Nonsquamous NSCLC with PD-L1 $<50 \%$ and low tumor mutational burden: pembrolizumab-pemetrexedplatinum combination seems to be a reasonable option.

4. Squamous NSCLC with PD-L1 $<50 \%$ and low tumor mutational burden: carboplatin-taxane-pembrolizumab might be the preferred treatment option (KeyNote-407).

- Among NSCLC patients with previous EGFR/ALK TKI treatment: carboplatin-paclitaxel-bevacizumabatezolizumab combination might be a reasonable option.

Moreover, for patients with unresectable stage III disease and no progression following chemoradiotherapy, durvalumab maintenance therapy improves outcomes and is the preferred treatment option. Results of additional studies are awaited to elucidate the potential role of PD-(L)1 inhibitors in the (neo)adjuvant treatment of resectable NSCLC.

Clinicians should be adequately trained in managing the complex side effect profile of these agents, and both radiologists and clinicians should be aware of the potential differences in response evaluation among solid tumor patients treated with these agents.

Financial \& competing interests disclosure

The authors have no relevant affiliations or financial involvement with any organization or entity with a financial interest in or financial conflict with the subject matter or materials discussed in the manuscript. This includes employment, consultancies, honoraria, stock ownership or options, expert testimony, grants or patents received or pending, or royalties.

No writing assistance was utilized in the production of this manuscript. 


\section{References}

1. Momtaz P, Postow MA. Immunologic checkpoints in cancer therapy: focus on the programmed death-1 (pd-1) receptor pathway. Pharmgenomics Pers. Med. 7, 357 (2014).

2. Rosenberg SA, Yang JC, Restifo NP. Cancer immunotherapy: moving beyond current vaccines. Nat. Med. 10(9), 909-915 (2004).

3. Banna GL, Passiglia F, Colonese F et al. Immune-checkpoint inhibitors in non-small cell lung cancer: a tool to improve patients' selection. Crit. Rev. Oncol. Hematol. 129, 27-39 (2018).

4. Brahmer J, Reckamp KL, Baas P et al. Nivolumab versus docetaxel in advanced squamous-cell non-small-cell lung cancer. N. Engl. J. Med. 373(2), 123-135 (2015).

5. Borghaei H, Paz-Ares L, Horn L et al. Nivolumab versus docetaxel in advanced nonsquamous non-small-cell lung cancer. N. Engl. J. Med. 373(17), 1627-1639 (2015).

6. Herbst RS, Baas P, Kim DW et al. Pembrolizumab versus docetaxel for previously treated, pd-11-positive, advanced non-small-cell lung cancer (keynote-010): A randomised controlled trial. Lancet 387(10027), 1540-1550 (2016).

7. Rittmeyer A, Barlesi F, Waterkamp D et al. Atezolizumab versus docetaxel in patients with previously treated non-small-cell lung cancer (oak): A phase III, open-label, multicentre randomised controlled trial. Lancet 389(10066), 255-265 (2017).

8. Abdel-Rahman O. Smoking and EGFRstatus may predict outcomes of advanced NSCLC treated with pd-(I) 1 inhibitors beyond first line: ameta-analysis. Clin. Respir. J. 12(5), 1809-1819 (2018).

9. Kim JH, Kim HS, Kim BJ. Prognostic value of smoking status in non-small-cell lung cancer patients treated with immune checkpoint inhibitors: a meta-analysis. Oncotarget 8(54), 93149-93155 (2017).

10. Reck M, Rodríguez-Abreu D, Robinson AG et al. Pembrolizumab versus chemotherapy for pd-11-positive non-small-cell lung cancer. $N$. Engl. J. Med. 375(19), 1823-1833 (2016).

11. Carbone DP, Reck M, Paz-Ares L et al. First-line nivolumab in stage iv or recurrent non-small-cell lung cancer. N. Engl. J. Med. 376(25), 2415-2426 (2017).

12. Steuer CE, Ramalingam SS. Tumor mutation burden: leading immunotherapy to the era of precision medicine? J. Clin. Oncol. 36(7), 631-632 (2018).

13. Hellmann MD, Ciuleanu T-E, Pluzanski A et al. Nivolumab plus ipilimumab in lung cancer with a high tumor mutational burden. $N$. Engl. J. Med. 378(22), 2093-2104 (2018).

14. Higgs BW, Morehouse CA, Brohawn PZ et al. 65pdhigh tumor mutational burden (tmb) and pd-11 have similar predictive utility in $21+$ nsclc patients (pts) treated with anti-pd-11 and anti-ctla-4. Ann. Oncol. 29(suppl_8), mdy269.063-mdy269.063 (2018).

15. Kim ES, Velcheti V, Mekhail T et al. Lba55primary efficacy results from b-f1 rst, a prospective Phase II trial evaluating blood-based tumour mutational burden (btmb) as a predictive biomarker for atezolizumab (atezo) in 11 non-small-cell lung cancer (NSCLC). Ann. Oncol. 29(suppl_8), mdy424.067-mdy424.067 (2018).

16. Gandhi L, Rodríguez-Abreu D, Gadgeel S et al. Pembrolizumab plus chemotherapy in metastatic non-small-cell lung cancer. N. Engl. J. Med. 378(22), 2078-2092 (2018).

17. Paz-Ares LG, Luft A, Tafreshi A et al. Phase IIIstudy of carboplatin-paclitaxel/nab-paclitaxel (chemo) with or without pembrolizumab (pembro) for patients (pts) with metastatic squamous (sq) non-small-cell lung cancer (nsclc). J. Clin. Oncol. 36(15_suppl), 105-105 (2018).

18. Socinski MA, Jotte RM, Cappuzzo F et al. Atezolizumab for first-line treatment of metastatic nonsquamous nsclc. N. Engl. J. Med. 378(24), 2288-2301 (2018).

19. Cappuzzo F, Mccleod M, Hussein M et al. Lba53impower130: progression-free survival (pfs) and safety analysis from a randomised Phase III study of carboplatin + nab-paclitaxel (cnp) with or without atezolizumab (atezo) as first-line (1l) therapy in advanced non-squamous nsclc. Ann. Oncol. 29(suppl_8), mdy424.065-mdy424.065 (2018).

20. Barlesi F, Nishio M, Cobo M et al. Lba54impower132: efficacy of atezolizumab (atezo) + carboplatin (carbo)/cisplatin (cis) + pemetrexed (pem) as 11 treatment in key subgroups with stage iv non-squamous non-small-cell lung cancer (NSCLC). Ann. Oncol. 29(suppl_8), mdy424.066-mdy424.066 (2018).

21. Lopes G, Wu Y-L, Kudaba I et al. Pembrolizumab (pembro) versus platinum-based chemotherapy (chemo) as first-line therapy for advanced/metastatic NSCLCwith a pd-11 tumor proportion score (tps) $\geq 1 \%$ : open-label, Phase III keynote-042 study. J. Clin. Oncol. 36(18_suppl) (2018).

22. Antonia SJ, Villegas A, Daniel D et al. Durvalumab after chemoradiotherapy in stage iii non-small-cell lung cancer. $N$. Engl. J. Med. 377(20), 1919-1929 (2017).

23. Faivre-Finn C, Spigel DR, Senan S et al. 1363oefficacy and safety evaluation based on time from completion of radiotherapy to randomization with durvalumab or placebo in pts from pacific. Ann. Oncol. 29(suppl_8), mdy291-mdy291 (2018).

24. Forde PM, Chaft JE, Smith KN et al. Neoadjuvant pd-1 blockade in resectable lung cancer. N. Engl. J. Med. 378(21), 1976-1986 (2018).

25. Cascone T, William WN, Weissferdt A et al. Lba49neoadjuvant nivolumab (n) or nivolumab plus ipilimumab (ni) for resectable non-small cell lung cancer (NSCLC). Ann. Oncol. 29(suppl_8), mdy424.059-mdy424.059 (2018). 
26. Abdel-Rahman O, Eltobgy M, Oweira H, Giryes A, Tekbas A, Decker M. Immune-related musculoskeletal toxicities among cancer patients treated with immune checkpoint inhibitors: a systematic review. Immunotherapy 9(14), 1175-1183 (2017).

27. Eltobgy M, Oweira $\mathrm{H}$, Petrausch U et al. Immune-related neurological toxicities among solid tumor patients treated with immune checkpoint inhibitors: a systematic review. Exp. Rev. Neurotherap. 17(7), 725-736 (2017).

28. Abdel-Rahman O, Fouad M. Risk of pneumonitis in cancer patients treated with immune checkpoint inhibitors: a meta-analysis. Ther. Adv. Respir. Dis. 10(3), 183-193 (2016).

29. Abdel-Rahman O. Nonconventional patterns of benefit of solid tumors treated with pd-(l)1 inhibitors: a systematic review. Immunotherapy 9(12), 995-1004 (2017).

30. Seymour L, Bogaerts J, Perrone A et al. Irecist: guidelines for response criteria for use in trials testing immunotherapeutics. Lancet Oncol. 18(3), e143-e152 (2017).

31. Abdel-Rahman O. Evaluation of efficacy and safety of different pembrolizumab dose/schedules in treatment of non-small-cell lung cancer and melanoma: a systematic review. Immunotherapy 8(12), 1383-1391 (2016).

32. Long GV, Tykodi SS, Schneider JG et al. Assessment of nivolumab exposure and clinical safety of $480 \mathrm{mg}$ every 4 weeks flat-dosing schedule in patients with cancer. Ann. Oncol. mdy408-mdy408 (2018). 
